\title{
Rules for Psychiatric Medications and Diagnoses
}

These rules should help children and families deal appropriately with psychiatric information, medication use and psychiatric diagnosis.

1. All medical matters are PRIVATE and no one needs to know or should know about medications or diagnosis except perhaps other family members.

2. Minors should NOT be allowed to handle their own medications. A parent or other adult should be in charge of medications at all times for anyone under 18 years of age. Adults need to handle all meds for safe-keeping, pouring, and watching the meds be swallowed. Youth should not be considered competent to handle their own medications anymore than they are competent to drink alcohol.

3. Children should be coached to ignore anyone who asks about medical matters. If pressed, a distortion is acceptable: "Everything is about allergies, and I cannot figure it out either." That should close the matter. Anything more will likely be used in a cruel way by peers, even best friends during those moments of incompatibility - and the best friend will feel worse about it than the patient will-do not give anyone ammunition-if you do, you will regret it. Medical matters are private family matters only.

4. Siblings should be told that medications, diagnosis, medical matters and going to the doctor are NOT fair game for use in arguments and fights. Medical matters are "off limits" during those moments of incompatibility.

5. A diagnosis is always confusing and puzzling. A diagnosis usually means that you have some kind of chemical imbalance that needs correcting by medications. Counseling also helps with the feelings, ideas, and thoughts that disrupt one's life. No matter what diagnostic name you are given, it has a good chance of going away if you take your medicine and follow directions. This is another reason not to take too seriously the diagnosis label because it may be temporary, and you may not have the problem for the rest of your life-so avoid making the diagnosis label a big deal outside of the doctor's office and home.

6. If someone tells you about their meds, diagnosis or doctor visits, be kind and supportive. Tell them that you have heard that if you take your medicine and follow the doctor's directions, the diagnosis is likely to go away. And tell them to stop telling everybody because some people will not understand and are likely to tease about it.

\section{Opinion}

Volume 5 Issue 2 - 2016

Dr. Samuel A Nigro M.D*

Retired, Assistant Clinical Professor Psychiatry, Case Western Reserve University School of Medicine, USA

*Corresponding author: Dr. Samuel A Nigro M.D, Retired, Assistant Clinical Professor Psychiatry, Case Western Reserve University School of Medicine, 2517 Guilford Road, Cleveland Heights, Ohio 44118, USA, Tel: 216 932-0575; Email:

sam@docnigro.com

Received: October 15, 2015 | Published: January 27, 2016 